\title{
EXISTENCE OF HOLOMORPHIC SECTIONS AND PERTURBATION OF POSITIVE LINE BUNDLES OVER $q$-CONCAVE MANIFOLDS
}

\author{
GEORGE MARINESCU
}

To Gennadi Henkin, in memoriam

\begin{abstract}
Universität zu Köln, Mathematisches Institut, Weyertal 86-90, 50931 Köln, Germany. Institute of Mathematics 'Simion Stoilow', Romanian Academy, Bucharest, Romania. E-mail: gmarines@math.uni-koeln.de
\end{abstract}

$\|$

Abstract

By using holomorphic Morse inequalities we prove that sufficiently small deformations of a pseudoconcave domain in a projective manifold is Moishezon.

\section{Introduction}

Let $X$ be a compact complex manifold with strongly pseudoconcave boundary. The question of projectively embedding $X$ has been studied in [1], 4], [5], 11], [13]. In particular the generalization of Kodaira's embedding theorem would give an intrinsic characterisation of projective pseudoconcave manifolds in terms of positive line bundles. Our model is the case when $X$ admits a positively embedded (i.e. with positive normal bundle) smooth compact divisor $Z$. By a rigidity theorem of Griffiths [13] we infer that global sections in high tensor powers of the the associated bundle $[Z]$ embed a small neighbourhood of $Z$ in the projective space. In particular $X$ has a maximal number of independent meromorphic functions.

Received May 15, 2016.

AMS Subject Classification: 32L10, 32F10, 32G05.

Key words and phrases: Deformation of complex structure, q-concave manifold, Moishezon manifold, holomorphic Morse inequalities. 
We will be concerned in the sequel with general positive line bundles. Since for $\operatorname{dim} X=2$ there exists examples of strongly pseudoconcave manifolds which possess positive line bundles but cannot be even compactified (see Andreotti-Siu [5]) we will restrict ourselves to the case $\operatorname{dim} X \geqslant 3$. For an analysis of the case $\operatorname{dim} X=2$, see Epstein-Henkin [11].

A first step towards the Kodaira embedding of $X$ is to find holomorphic sections in the tensor powers $E^{k}$ of a positive line bundle $E$ over $X$. We will prove an existence criterion giving a lower bound for $\operatorname{dim} H^{0}\left(X, E^{k}\right)$ in terms of geometric data such as the Levi form of $\partial X$ and the curvature of $E$. As a corollary we see that, roughly speaking, if the volume of $X$ in the metric $\imath \mathbf{c}(E)$ exceeds the volume of $\partial X$ times a constant expressing the size of the Levi form and of the curvature $\imath \mathbf{c}(E)$ near the boundary, the ring $\bigoplus_{k \geqslant 0} H^{0}\left(X, E^{k}\right)$ contains local coordinates for each point outside a proper analytic set of $X$. Since for $\operatorname{dim} X \geqslant 3$ there exists a compactification $\hat{X}$ which contains $X$ as an open set we deduce that $\hat{X}$ is Moishezon. We remind that by an important theorem of Moishezon a compact complex manifold with a maximal number of independent meromorphic functions (this being the definition of Moishezon manifolds) is not far from being projective. Namely, there exists a proper projective modification of $\hat{X}$. This implies that modifiying $X$ along a proper analytic set (which may cut the boundary) we obtain an open set in a projective manifold. Note that the main result of Andreotti-Tomassini [4] (see also [5]) says that if $\bigoplus_{k \geqslant 0} H^{0}\left(X, E^{k}\right)$ separates points and gives local coordinates on $X$ there exists a projective manifold $\hat{X}$ containing $X$ as an open set. Our aim is to find geometric conditions which imply the hypothesis in Andreotti-Tomassini theorem.

In the pesent paper we work actually with a more general class of manifolds, namely $q$-concave manifolds. In this terminology, strongly pseudoconcave manifolds correspond to 1-concave manifolds. As application of the existence theorem we prove a stability property for certain $q$-concave manifolds. Let us consider the complement $X$ of a sufficiently small neighbourhood of a submanifold of codimension $\geqslant 3$ in a projective manifold. Assume that we perform a small perturbation of the complex stucture of $X$ such that along a (not necessaraly compact) smooth divisor the structure remains unchanged. Then the resulting manifold still has a maximal number of meromorphic functions. If moreover the canonical bundle is positive, any small perturbation suffices for the result to hold. 


\section{Description of the Results}

In this paper we shall be concerned with pseudoconcave (for short concave) complex manifolds. We understand concavity in the sense of AndreottiGrauert [2]. A manifold $X$ of dimension $n$ is called $q$-concave if there exists a smooth function $\varphi: X \longrightarrow(a, b]$ where $a=\inf \varphi \in\{-\infty\} \cup \mathbb{R}, b \in \mathbb{R}$, such that $X_{c}:=\{\varphi>c\} \Subset X$ for all $c \in(a, b]$ and $\imath \partial \bar{\partial} \varphi$ has at least $n-q+1$ positive eigenvalues outside an exceptional compact set $K$. The prime examples of such manifolds arise as complements of complex submanifolds of compact manifolds. More precisely, let $M$ be a compact complex manifold and $A \subset M$ of dimension $q$. Then $M \backslash A$ is $(q+1)$-concave (see $\S 4$.). It is well known (see [1]) that for a $q$-concave manifold $X(q \leqslant n-1)$ the transcedence degree $\operatorname{deg} \operatorname{tr} \mathcal{K}(X)$ of the meromorphic function field is at most the complex dimension of $X$. In analogy to the corresponding notion for compact manifolds we say that a $q$-concave manifold is Moishezon if $\operatorname{deg} \operatorname{tr} \mathcal{K}(X)=\operatorname{dim}_{\mathbb{C}} X$.

Let us consider now a projective manifold $M$, a submanifold $A \subset M$ and the concave manifold $X:=M \backslash A$. Our aim is to study to what extent small deformations of the sublevel sets $X_{c}$ for small values of $c>\inf \varphi$ (i.e. for $X_{c}$ close to $X$ ) give rise to concave Moishezon manifolds. As a matter of fact we may consider small neighbourhoods $V$ of $A$, which means that $X_{c} \subset M \backslash V$ for small $c>\inf \varphi$. Then $M \backslash V$ is pseudoconcave in the sense of Andreotti and the notion of Moishezon manifold still makes sense (see [1]).

Stability Theorem. Let $M$ be a compact projective manifold and let $Z$ be an ample smooth divisor. Let $A \subset M$ be a complex submanifold of codimension at least 3. Then for any sufficiently small neighbourhood $V$ of $A$ and for any sufficiently small deformation of the complex structure of $M \backslash V$ leaving $T(Z)$ invariant, the manifold $M \backslash V$ with the new structure is a pseudoconcave Moishezon manifold. If the canonical bundle $K_{M}$ is positive, the statement holds for any small enough perturbation.

Let us note that the sublevel sets $X_{c}$ are also $q$-concave manifolds if $K \Subset X_{c}$. Our method is based on $L^{2}$ estimates for $(0,1)$-forms on $X_{c}$ and for this reason we need at least 3 positive eigenvalues for $\imath \partial \bar{\partial} \varphi$, that is $\operatorname{codim} A=n-q \geqslant 3$. While this condition may seem technical we can 
explain it as follows. The existence of $L^{2}$ estimates for $(0,1)$-forms imply the finiteness of the first cohomology group $H^{1}(X, F)$ for holomorphic vector bundles $F$ over $X$. By the Andreotti-Grauert theory [2, 3, 15] we have $\operatorname{dim} H^{p}(X, F)<\infty$ for $p \leqslant n-(q+1)-1=n-q-2$ but $\operatorname{dim} H^{p}(X, F)=\infty$ for $p=n-q-1$ if the Levi form is non-degenerate on the boundary (see [3, Théorème 2]). Therefore we have to impose $n-q-1>1$ i.e. $n-q>2$.

An immediate consequence is the following.

Corollary 2.1. Let $M$ be a compact projective manifold and let let $Z$ be an ample smooth divisor. Let $A \subset M$ be a complex submanifold of codimension at least 3. Then for any sufficiently small neighbourhood $V$ of $A$ and for any deformation of the complex structure of $M$ which is sufficiently small on $M \backslash V$ and leaves $T(Z)$ invariant, the manifold $M$ with the new structure is Moishezon.

In order to prove the Stability Theorem we need a differential geometric criterion for a $q$-concave manifold to be Moishezon. For compact manifolds the type of results we need were proved by Siu [27] and Demailly [9]. They derive asymptotic Morse inequalities for the cohomology groups with values in the tensor powers of a holomorphic line bundle. For non-compact manifolds the Morse inequalities were used by Nadel-Tsuji [24] to prove the quasi-projectivity of very strongly $(n-2)$-concave manifolds of dimension $n$ which possess a complete Kähler metric with Ric $\omega<0$ and whose universal covering is Stein. In [23], 28] we considered Morse inequalities for general 1-concave manifolds with application to the deformation of the complex structure of compact complex spaces with isolated singularities. In the sequel we study $q$-concave manifolds and give an estimate from below of the dimension of the space of holomorphic sections with values in a positive line bundle (see the Existence Criterion below). An important feature of our estimate is the presence of a negative boundary term which expresses the obstruction to finding holomorphic sections.

We need some preparations and notations in order to state the result. Let $X$ be a $q$-concave manifold with exhaustion function $\varphi$. If $\partial X_{c}$ is smooth the Levi form of $\partial X_{c}$ has at least $n-q-1$ negative eigenvalues (since the defining function for $X_{c}$ is $c-\varphi$ ). Therefore the following setting may be considered. 
Let $D \Subset X$ be a smooth domain in a complex manifold $X$ such that the Levi form of $\partial D$ has at least 2 negative eigenvalues. Then we can choose a defining function $\varphi$ for $D$ which is smooth on $\bar{D}, D=\{\varphi<0\}$ and $\partial \bar{\partial} \varphi$ has at least 3 negative eigenvalues. We can in fact modify a defining function in order to get an extra negative eigenvalue in the complex normal direction to $\partial D$. In the following we keep the function $\varphi$ fixed.

We introduce a hermitian metric $\omega=\omega_{\varphi}$ in the neighbourhood of $\bar{D}$ such that in a neighbourhood $V$ of $\partial D$ the following property holds:

Property 2.2. The first 3 eigenvalues of $\imath \partial \bar{\partial} \varphi$ with respect to $\omega$ are at most $-2 n+3$ and all others are at most 1 .

Finally set $d S_{E}$ for the volume form of $\partial D$ in the induced metric from $\imath \mathbf{c}(E)$ and $|d \varphi|_{E}$ for the norm of $d \varphi$ in the metric associated to $\imath \mathbf{c}(E)$.

We can state the estimate for the dimension of the space holomorphic sections on the concave domain $D$.

Existence Criterion. Let $D \Subset X$ be a smooth domain in a complex manifold $X$ such that the Levi form of $\partial D$ possesses at least 2 negative eigenvalues. Let $E$ be a holomorphic line bundle on $X$ which is assumed to be positive on a neighbourhood of $\bar{D}$. Then

$$
\liminf _{k \longrightarrow \infty} k^{-n} \operatorname{dim} H^{0}\left(D, E^{k}\right) \geqslant \int_{D}\left(\frac{\imath}{2 \pi} \mathbf{c}(E)\right)^{n}-C(\varphi, E) \int_{\partial D} \frac{d S_{E}}{|d \varphi|_{E}}
$$

The constant $C(\varphi, E)$ depends explicitely on the curvature of $E$ and on the Levi form $\imath \partial \bar{\partial} \varphi$ (cf. (3.1)).

Berman [7] obtained a similar estimate where the boundary integral involves the Levi form. Estimates of type (2.1) fall under the name holomorphic Morse inequalities and were introduced by Demailly [9], see also [21]. In the case of manifolds with boundary they are related to Morse inequalities for the Kohn-Rossi cohomology on a CR manifold, see [12, 17, 18, 19]. For Morse Inequalities on pseudoconvex domains see [8, 22] and [21, §3.5].

\section{Proof of the Existence Criterion}

A familiar method of producing holomorphic sections in a positive bundle $E$ is the use of $L^{2}$ estimates for $\bar{\partial}$ of Andreotti-Vesentini and Hörmander 
(see e.g. 6], [10] and [25]). The $L^{2}$ estimates may be established equally in the case of pseudoconvex and pseudoconcave manifolds by introducing 'weights' (i.e. changing the hermitian metric on the bundle) that reflect the convexity or concavity of the manifold. The problem is that, for pseudoconcave manifolds the positivity is lost by this procedure (in contrast to the pseudoconvex case). There is however a strategy of finding holomorphic sections in non-positive hermitian bundles which has been introduced by Siu [27] for semipositive line bundles and then generalized by Demailly [9] into his asymptotic Morse inequalities. The main ingredient is a Weyl type formula describing the semiclassical behaviour of the $\bar{\partial}$-laplacian on the tensor powers $E^{k}$. The first applications for non-compact manifolds appear in Nadel-Tsuji [24] and Bouche [8].

We proceed as follows. In a first instance we find a good $L^{2}$-estimate for the $(0,1)$-forms with values in $E^{k}$. Then following Bouche [8] we compare the spectrum of the Laplace operator on $D$ (for a complete metric) with the spectrum of the Dirichlet problem over a smaller domain $D(\varepsilon / 2)$ which is a set of points of $D$ at distance less than $\sqrt{\varepsilon / 2}$ times a certain constant from $\partial D$ (see (3.11) for the precise definition). On $D(\varepsilon / 2)$ we can use Demailly's spectral formula and get a lower bound for the dimension of the space of sections in $E^{k}$ for large $k$. We shall need the full strength of Demailly's result since the curvature of the changed metric has negative eigenvalues. In the last step we apply the results to metrics which approximate the positive metric on $E$ in the interior of the manifold. In the process of approximation the set where the curvature has a negative part concentrates to the boundary $\partial D$ and is responsible for the negative boundary term in the final estimate of the Existence Criterion.

We begin by setting some notations and defining the constant $C(\varphi, E)$.

Let $\eta$ a hermitian metric on $X, \Phi$ a real $(1,1)$-form and $K$ a compact set in $X$. We set:

$$
M_{\eta}(\Phi, K)=\sup _{x \in K} \sup _{v \in T_{x} X \backslash\{0\}} \frac{\Phi(v, v)}{\eta(v, v)},
$$

the supremum over $K$ of the highest eigenvalue of $\Phi$ with respect to $\eta$. In hindsight to our previous situation denote:

$$
M_{E}(\varphi)=M_{\imath \mathbf{c}(E)}(\imath \partial \bar{\partial} \varphi, \bar{D})
$$




$$
\begin{aligned}
& M_{E}(-\varphi)=M_{\imath \mathbf{c}(E)}(-\imath \partial \bar{\partial} \varphi, \bar{D}) \\
& M_{\omega}(E)=M_{\omega}(\imath \mathbf{c}(E), \bar{D}) \\
& M_{\omega}^{\prime}(E)=1+2(n-1) M_{\omega}(\imath \mathbf{c}(E), \bar{D}) \\
& M_{E}(\partial \varphi)=M_{\imath \mathbf{c}(E)}(\imath \partial \varphi \wedge \bar{\partial} \varphi, \partial D)
\end{aligned}
$$

which represent the relative size of the respective $(1,1)$-forms. We also put:

$$
\begin{aligned}
& C_{1}=\sqrt{2 M_{E}(-\varphi) M_{\omega}^{\prime}(E)}-1 \\
& C_{2}=2 M_{E}(-\varphi) M_{\omega}^{\prime}(E)-1 \\
& C_{3}=2 M_{E}(\varphi) M_{\omega}^{\prime}(E)+1 \\
& C_{4}=2 M_{\omega}^{\prime}(E) M_{E}(\partial \varphi)
\end{aligned}
$$

The definition of $C(\varphi, E)$ is then

$$
C(\varphi, E)=(2 \pi)^{-n} C_{1} C_{2} C_{3}^{n-2} C_{4}
$$

Let $\gamma_{1} \leqslant \gamma_{2} \leqslant \cdots \leqslant \gamma_{n}$ be the eigenvalues of $\imath \partial \bar{\partial} \varphi$ with respect to $\omega$. We have chosen $\omega$ such that (see Property 2.2) in a neighbourhood $V$ of $\partial D$,

$$
\begin{aligned}
& \gamma_{1} \leqslant \gamma_{2} \leqslant \gamma_{3} \leqslant-2 n+3 \\
& \gamma_{n} \leqslant 1
\end{aligned}
$$

Let $\chi:(-\infty, 0) \longrightarrow \mathbb{R}, \chi(t)=t^{-2}$. We consider the complete metric:

$$
\omega_{0}=\omega+\chi(\varphi) \partial \varphi \wedge \bar{\partial} \varphi
$$

which grows as $\varphi^{-2}$ in the normal direction to $\partial D$. Along the fibers of $E$ we introduce the metric:

$$
h_{\varepsilon}=h \exp \left(-\varepsilon \int_{\inf \varphi}^{\varphi} \chi(t) d t\right)
$$

where $h$ is the given metric on $E$ (for which $\imath \mathbf{c}(E)$ is positive). The curvature of $h_{\varepsilon}$ is

$$
\imath \mathbf{c}\left(E, h_{\varepsilon}\right)=\imath \mathbf{c}(E)+\imath \varepsilon \chi(\varphi) \partial \bar{\partial} \varphi+\imath \varepsilon \chi^{\prime}(\varphi) \partial \varphi \wedge \bar{\partial} \varphi
$$

We evaluate the eigenvalues of $\imath \mathbf{c}\left(E, h_{\varepsilon}\right)$ with respect to $\omega_{0}$ with the goal to apply the Bochner-Kodaira formula. Denote by $\gamma_{1}^{0} \leqslant \gamma_{2}^{0} \leqslant \cdots \leqslant \gamma_{n}^{0}$ the 
eigenvalues of $\imath \partial \bar{\partial} \varphi$ and $\Gamma_{1}^{\varepsilon} \leqslant \Gamma_{2}^{\varepsilon} \leqslant \cdots \leqslant \Gamma_{n}^{\varepsilon}$ the eigenvalues of $\imath \varepsilon \chi(\varphi) \partial \bar{\partial} \varphi+$ $\imath \varepsilon \chi^{\prime}(\varphi) \partial \varphi \wedge \bar{\partial} \varphi$ with respect to $\omega_{0}$. The minimum-maximum principle yields

$$
\begin{array}{ll}
\gamma_{1} \leqslant \gamma_{1}^{0} \leqslant \gamma_{2} \leqslant \gamma_{2}^{0} \leqslant \gamma_{3} \leqslant-2 n+3 & \text { by (3.2a) }, \\
\gamma_{3}^{0}<0 & \text { since } \gamma_{3}<0, \\
\gamma_{j}^{0} \leqslant \max \left\{\gamma_{n}, 0\right\} \leqslant 1 \quad \text { for } 4 \leqslant j \leqslant n, & \text { by }(3.2 \mathrm{~b}) .
\end{array}
$$

on $V$. It is also easy to see that the highest eigenvalue of $\imath \chi^{\prime}(\varphi) \partial \varphi \wedge \bar{\partial} \varphi$ with respect to $\omega_{0}$ satisfies

$$
\sup _{v \in T_{x} X \backslash\{0\}} \frac{\imath \chi^{\prime}(\varphi) \partial \varphi \wedge \bar{\partial} \varphi(v, v)}{\omega_{0}(v, v)} \leqslant \chi(\varphi), \quad \text { for all } x \in D .
$$

By (3.6) we have

$$
\Gamma_{j}^{\varepsilon} \leqslant \varepsilon \chi(\varphi)\left(\gamma_{j}^{0}+1\right)
$$

and therefore,

$$
\begin{aligned}
& \Gamma_{1}^{\varepsilon} \leqslant \Gamma_{2}^{\varepsilon} \leqslant(-2 n+4) \varepsilon \chi(\varphi) \quad \text { by (3.5a) } \text {, } \\
& \Gamma_{3}^{\varepsilon} \leqslant \varepsilon \chi(\varphi) \quad \text { by }(3.5 \mathrm{~b}) \text {, } \\
& \Gamma_{j}^{\varepsilon} \leqslant 2 \varepsilon \chi(\varphi) \text { for } 4 \leqslant j \leqslant n, \quad \text { by (3.5c). }
\end{aligned}
$$

Summing up we obtain

$$
\Gamma_{2}^{\varepsilon}+\cdots+\Gamma_{n}^{\varepsilon} \leqslant-\varepsilon \chi(\varphi) .
$$

This sum will appear in the Bochner-Kodaira formula and carries the information about the concavity of $D$.

We also have to estimate the eigenvalues of $\imath \mathbf{c}(E)$ with respect to $\omega_{0}$. We denote by $\alpha_{1} \leqslant \alpha_{2} \leqslant \cdots \leqslant \alpha_{n}$ the eigenvalues of $\imath \mathbf{c}(E)$ with respect to $\omega$ and by $\alpha_{1}^{0} \leqslant \alpha_{2}^{0} \leqslant \cdots \leqslant \alpha_{n}^{0}$ the eigenvalues of $\imath \mathbf{c}(E)$ with respect to $\omega_{0}$. It is straightforward that

$$
\alpha_{n}^{0} \leqslant \alpha_{n} \leqslant M_{\omega}(E)<\infty \quad \text { on } V .
$$

Since the torsion operator of $\omega_{0}$ with respect to $\omega_{0}$ are bounded by a constant $A>0$ (depending only on $\omega_{0}$ ), the Bochner-Kodaira formula 
assumes the following form (see e.g. [9], [25]):

$$
\begin{aligned}
& \frac{3}{2}\left(\|\bar{\partial} u\|^{2}+\left\|\bar{\partial}^{*} u\right\|^{2}\right) \\
& \quad \geqslant \int_{D}\left[-k\left(\Gamma_{2}^{\varepsilon}+\cdots+\Gamma_{n}^{\varepsilon}\right)-k\left(\alpha_{2}^{0}+\cdots+\alpha_{n}^{0}\right)-A \chi(\varphi)\right]|u|^{2} d V
\end{aligned}
$$

for any compactly supported $(0,1)$-form in $D$ with values in $E^{k}$. The volume form is taken with respect to $\omega_{0}$ and the norms are with respect to $\omega_{0}$ on $D$ and $h_{\varepsilon}$ on $E$. The inequalities (3.7), (3.8) and (3.9) entail

$$
\frac{3}{2}\left(\|\bar{\partial} u\|^{2}+\left\|\bar{\partial}^{*} u\right\|^{2}\right) \geqslant \int_{D}\left[-k(n-1) M_{\omega}(E)+k \varepsilon \chi(\varphi)-A \chi(\varphi)\right]|u|^{2} d V
$$

for any compactly supported $(0,1)$-form in $D$ with values in $E^{k}$ and support in $V$. We use now the term $k \varepsilon \chi(\varphi)$ to absorb the negative terms in the lefthand side of (3.10). We introduce the following notation:

$$
D(\varepsilon)=\left\{x \in D: \varphi(x)<-\sqrt{\varepsilon / M_{\omega}^{\prime}(E)}\right\} .
$$

We may assume that $V$ contains the set $\complement D(\varepsilon)$ (for $\varepsilon$ small enough). In the set $\complement D(\varepsilon)$ we have $\varepsilon \chi(\varphi) \geqslant M_{\omega}^{\prime}(E)$ and if we choose $k \geqslant 2 A \varepsilon^{-1}$ we get

$$
-k(n-1) M_{\omega}(E)+k \varepsilon \chi(\varphi)-A \chi(\varphi) \geqslant \frac{k}{2}
$$

so that (3.10) yields

$$
3\left(\|\bar{\partial} u\|^{2}+\left\|\bar{\partial}^{*} u\right\|^{2}\right) \geqslant k \int_{D}|u|^{2} d V, \operatorname{supp} u \Subset \complement D(\varepsilon), k \geqslant 2 A \varepsilon^{-1}
$$

Since the metric $\omega_{0}$ is complete we deduce that (3.12) holds true for any $(0,1)$-form $u \in \operatorname{Dom} \bar{\partial} \cap \operatorname{Dom} \bar{\partial}^{*}$ with support in $\complement D(\varepsilon)$ (by the density lemma of Andreotti-Vesentini [6]).

Estimate (3.12) is crucial for our purpose. In Nadel-Tsuji [24] and [23] the spectral formula of Demailly was used to obtain a lower bound for the dimension of the space of holomorphic sections of bundles over pseudoconcave manifolds. After having established (3.12) we should just follow the same lines. We give the details since we need the precise output to be able to make $\varepsilon \longrightarrow 0$. 
By following Demailly [9] we reduce the problem to estimating the size of certain spectral spaces of the $\bar{\partial}$-laplacian. Let us consider the operator $\frac{1}{k} \Delta_{k, \varepsilon}^{\prime \prime}$ where $\Delta_{k, \varepsilon}^{\prime \prime}=\bar{\partial} \bar{\partial}^{*}+\bar{\partial}^{*} \bar{\partial}$ is the Laplace-Beltrami operator acting on $(0, j)$-forms with values in $E^{k}$ over $D$. The metrics used to construct the adjoint $\bar{\partial}^{*}$ are $\omega_{0}$ and $h_{\varepsilon}$. Let $Q_{k, \varepsilon}$ be the quadratic form associated to $\frac{1}{k} \Delta_{k, \varepsilon}^{\prime \prime}$, that is, $Q_{k, \varepsilon}(u)=\frac{1}{k}\left(\|\bar{\partial} u\|^{2}+\left\|\bar{\partial}^{*} u\right\|^{2}\right)$. We denote by $E^{j}\left(\lambda, \frac{1}{k} \Delta_{k, \varepsilon}^{\prime \prime}\right)$ the spectral projectors and by

$$
\begin{aligned}
L^{j}\left(\lambda, \frac{1}{k} \Delta_{k, \varepsilon}^{\prime \prime}\right) & =\operatorname{Ran} E^{j}\left(\lambda, \frac{1}{k} \Delta_{k, \varepsilon}^{\prime \prime}\right), \\
N^{j}\left(\lambda, \frac{1}{k} \Delta_{k, \varepsilon}^{\prime \prime}\right) & =\operatorname{dim} L^{j}\left(\lambda, \frac{1}{k} \Delta_{k, \varepsilon}^{\prime \prime}\right),
\end{aligned}
$$

the spectral space and the counting function for the spectrum of $\frac{1}{k} \Delta_{k, \varepsilon}^{\prime \prime}$ on $(0, j)$-forms.

Lemma 3.1. For any $\lambda \geqslant 0$ and $k \geqslant 0$,

$$
\operatorname{dim} H^{0}\left(D, E^{k} \otimes K_{X}\right)+N^{1}\left(\lambda, \frac{1}{k} \Delta_{k, \varepsilon}^{\prime \prime}\right) \geqslant N^{0}\left(\lambda, \frac{1}{k} \Delta_{k, \varepsilon}^{\prime \prime}\right) .
$$

Proof. Since $\frac{1}{k} \Delta_{k, \varepsilon}^{\prime \prime}$ commutes with $\bar{\partial}$ it follows that the spectral projections of $\frac{1}{k} \Delta_{k, \varepsilon}^{\prime \prime}$ commute with $\bar{\partial}$ too, showing thus $\bar{\partial} L^{0}\left(\lambda, \frac{1}{k} \Delta_{k, \varepsilon}^{\prime \prime}\right) \subset L^{1}\left(\lambda, \frac{1}{k} \Delta_{k, \varepsilon}^{\prime \prime}\right)$ and therefore we have the bounded operator $\bar{\partial}_{\lambda}: L^{0}\left(\lambda, \frac{1}{k} \Delta_{k, \varepsilon}^{\prime \prime}\right) \longrightarrow L^{1}(\lambda$, $\left.\frac{1}{k} \Delta_{k, \varepsilon}^{\prime \prime}\right)$ where $\bar{\partial}_{\lambda}$ denotes the restriction of $\bar{\partial}$ (by the definition of $L^{0}\left(\lambda, \frac{1}{k} \Delta_{k, \varepsilon}^{\prime \prime}\right)$, $\bar{\partial}_{\lambda}$ is bounded by $k \lambda$ ). The assertion is a consequence of the following obvious relations:

$$
\begin{aligned}
N^{0}\left(\lambda, \frac{1}{k} \Delta_{k, \varepsilon}^{\prime \prime}\right) & =\operatorname{dim} \operatorname{ker} \bar{\partial}_{\lambda}+\operatorname{dim} \operatorname{Ran} \bar{\partial}_{\lambda}, \\
\operatorname{dim} \operatorname{Ran} \bar{\partial}_{\lambda} & \leqslant N^{1}\left(\lambda, \frac{1}{k} \Delta_{k, \varepsilon}^{\prime \prime}\right), \\
\operatorname{dim} \operatorname{ker} \bar{\partial}_{\lambda} & \leqslant \operatorname{dim} H^{0}\left(D, E^{k} \otimes K_{X}\right),
\end{aligned}
$$

where the last line follows from the fact that the kernel of $\bar{\partial}_{\lambda}$ consits of holomorphic sections.

By the previous lemma we have to estimate $N^{1}\left(\lambda, \frac{1}{k} \Delta_{k, \varepsilon}^{\prime \prime}\right)$ from above and then $N^{0}\left(\lambda, \frac{1}{k} \Delta_{k, \varepsilon}^{\prime \prime}\right)$ from below.

In the next Lemma we show that the essential spectrum of $\frac{1}{k} \Delta_{k, \varepsilon}^{\prime \prime}$ on $(0,1)$-forms does not contain the open interval $(0,1 / 24)$ and we can compare the counting function on this interval with the counting function of 
the same operator considered with Dirichlet boundary conditions on the domain $D(\varepsilon / 2)$ (introduced in (3.11) ) and denoted $\frac{1}{k} \Delta_{k, \varepsilon}^{\prime \prime} \uparrow_{D(\varepsilon / 2)}$. In particular $N^{1}\left(\lambda, \frac{1}{k} \Delta_{k, \varepsilon}^{\prime \prime}\right)$ is finite dimensional for $\lambda<1 / 24$. If $E^{j}\left(\mu, \frac{1}{k} \Delta_{k, \varepsilon}^{\prime \prime} \uparrow_{D(\varepsilon / 2)}\right)$ denote the spectral projectors of $\frac{1}{k} \Delta_{k, \varepsilon}^{\prime \prime} \uparrow_{D(\varepsilon / 2)}$ on $(0, j)$-forms we let

$$
\begin{aligned}
L^{j}\left(\mu, \frac{1}{k} \Delta_{k, \varepsilon}^{\prime \prime}\left\lceil_{D(\varepsilon / 2)}\right)\right. & =\operatorname{Ran} E^{j}\left(\mu, \frac{1}{k} \Delta_{k, \varepsilon}^{\prime \prime} \Upsilon_{D(\varepsilon / 2)}\right), \\
N^{j}\left(\mu, \frac{1}{k} \Delta_{k, \varepsilon}^{\prime \prime}\left\lceil_{D(\varepsilon / 2)}\right)\right. & =\operatorname{dim} L^{j}\left(\mu, \frac{1}{k} \Delta_{k, \varepsilon}^{\prime \prime}\left\lceil_{D(\varepsilon / 2)}\right),\right.
\end{aligned}
$$

be the spectral spaces and the spectrum distribution function. For the following lemma compare [16, Lemma 2.1] and [8, Théorème 2.1].

Lemma 3.2. For $k$ sufficiently large the operator $\frac{1}{k} \Delta_{k, \varepsilon}^{\prime \prime}$ on $(0,1)$-forms has discrete spectrum in $(0,1 / 24)$ and

$$
N^{1}\left(\lambda, \frac{1}{k} \Delta_{k, \varepsilon}^{\prime \prime}\right) \leqslant N^{1}\left(24 \lambda+16 C_{\varepsilon} k^{-1}, \frac{1}{k} \Delta_{k, \varepsilon}^{\prime \prime}\left\lceil_{D(\varepsilon / 2)}\right)\right.
$$

for $\lambda \in(0, \varepsilon / 2)$, where $C_{\varepsilon}$ is a constant independent of $k$.

Proof. Let $\rho_{\varepsilon} \in \mathcal{C}^{\infty}(D)$ such that $\rho_{\varepsilon}=0$ on a closed neighbourhood of $D(\varepsilon)$ and $\rho_{\varepsilon}=1$ on $\complement D(\varepsilon / 2)$. Let $u \in \operatorname{Dom}\left(Q_{k, \varepsilon}\right)=\operatorname{Dom} \bar{\partial} \cap \operatorname{Dom} \bar{\partial}^{*}$ be a $(0,1)$-form with values in $E^{k}$. Then $\rho_{\varepsilon} u$ has support in $\complement D(\varepsilon)$ and for $\rho_{\varepsilon} u$ we can apply (3.12). We also need the following simple estimate. Denote $C_{\varepsilon}=6 \sup \left|d \rho_{\varepsilon}\right|^{2}<\infty$. The constant depends on $\varepsilon$ (which is fixed) but not on $k$. Then

$$
Q_{k, \varepsilon}\left(\rho_{\varepsilon} u\right) \leqslant \frac{3}{2} Q_{k, \varepsilon}(u)+C_{\varepsilon} k^{-1}\|u\|^{2} .
$$

Using $\|u\|^{2} \leqslant 2\left(\left\|\rho_{\varepsilon} u\right\|^{2}+\left\|\left(1-\rho_{\varepsilon}\right) u\right\|^{2}\right)$ and then applying (3.12) to $\rho_{\varepsilon} u$ in conjunction with (3.13) we obtain:

$$
\|u\|^{2} \leqslant 12 Q_{k, \varepsilon}(u)+8 \int_{D(\varepsilon / 2)}|(1-\rho) u|^{2}, \quad k \geqslant \max \left\{2 A \varepsilon^{-1}, 4 C_{\varepsilon}\right\}
$$

for any $u \in \operatorname{Dom}\left(Q_{k, \varepsilon}\right)$. From relation (3.14) we infer that the spectral spaces corresponding to the lower part of the spectrum of $\frac{1}{k} \Delta_{k, \varepsilon}^{\prime \prime}$ on $(0,1)-$ forms can be injected into the spectral spaces of $\frac{1}{k} \Delta_{k, \varepsilon}^{\prime \prime} \uparrow_{D(\varepsilon / 2)}$ which correspond to the Dirichlet problem on $D(\varepsilon / 2)$. Namely, for $\lambda<1 / 24$, the morphism

$$
L^{1}\left(\lambda \frac{1}{k} \Delta_{k, \varepsilon}^{\prime \prime}\right) \longrightarrow L^{1}\left(24 \lambda+16 C_{\varepsilon} k^{-1}, \frac{1}{k} \Delta_{k, \varepsilon}^{\prime \prime}\left\lceil_{D(\varepsilon / 2)}\right)\right.
$$




$$
u \longmapsto E^{1}\left(24 \lambda+16 C_{\varepsilon} k^{-1}, \frac{1}{k} \Delta_{k, \varepsilon}^{\prime \prime} \uparrow_{D(\varepsilon / 2)}\right)\left(1-\rho_{\varepsilon}\right) u
$$

is injective. In order to prove the injectivity we choose $u \in L^{1}\left(\lambda, \frac{1}{k} \Delta_{k, \varepsilon}^{\prime \prime}\right)$, $\lambda<1 / 24$ to the effect that $Q_{k, \varepsilon}(u) \leqslant \lambda\|u\|^{2} \leqslant(1 / 24)\|u\|^{2}$. Plugging this relation in (3.14) we get

$$
\|u\|^{2} \leqslant 16 \int_{D(\varepsilon / 2)}|(1-\rho) u|^{2}, \quad u \in L^{1}\left(\lambda, \frac{1}{k} \Delta_{k, \varepsilon}^{\prime \prime}\right), \quad \lambda<1 / 24 .
$$

Let us denote by $Q_{k, D(\varepsilon / 2)}$ the quadratic form of $\frac{1}{k} \Delta_{k, \varepsilon}^{\prime \prime} \uparrow_{D(\varepsilon / 2)}$. Then by (3.13) and (3.15),

$$
\begin{aligned}
Q_{k, D(\varepsilon / 2)}((1-\rho) u) & \leqslant \frac{3}{2} Q_{k, \varepsilon}(u)+C k^{-1}\|u\|^{2} \\
& \leqslant\left(24 \lambda+16 C_{\varepsilon} k^{-1}\right) \int_{D(\varepsilon / 2)}|(1-\rho) u|^{2} .
\end{aligned}
$$

Thus $E^{1}\left(24 \lambda+16 C_{\varepsilon} k^{-1}, \frac{1}{k} \Delta_{k, \varepsilon}^{\prime \prime}\left\lceil_{D(\varepsilon / 2)}\right)(1-\rho) u=0\right.$ entails $(1-\rho) u=0$ so that $u=0$ by (3.15).

We obtain now a lower estimate for $N^{0}\left(\lambda, \frac{1}{k} \Delta_{k, \varepsilon}^{\prime \prime}\right)$.

Lemma 3.3. For $\lambda<1 / 24$ and sufficiently large $k$ the following relation holds :

$$
N^{0}\left(\lambda, \frac{1}{k} \Delta_{k, \varepsilon}^{\prime \prime}\right) \geqslant N^{0}\left(\lambda, \frac{1}{k} \Delta_{k, \varepsilon}^{\prime \prime} \Upsilon_{D(\varepsilon / 2)}\right)
$$

Proof. It is straightforward to show that the $L^{2}$ estimate (3.12) holds also for $(0,0)$-forms. Therefore by repeating the proof of Lemma 2.2 we see that the spectrum of $\frac{1}{k} \Delta_{k, \varepsilon}^{\prime \prime}$ on $(0,0)$-forms is discrete in the interval $(0,1 / 24)$. We may now apply the min-max principle to the operators $\frac{1}{k} \Delta_{k, \varepsilon}^{\prime \prime}$ and $\frac{1}{k} \Delta_{k, \varepsilon}^{\prime \prime} \uparrow_{D(\varepsilon / 2)}$ on this interval. Since $\operatorname{Dom}\left(Q_{k, \varepsilon}\right) \supset \operatorname{Dom}\left(Q_{k, D(\varepsilon / 2)}\right)$ the desired result follows immediatly.

The asymptotic behaviour of the spectrum distribution function for the Dirichlet problem has been determined explicitely by Demailly [9]. Since for $\varepsilon$ small enough $\partial D(\varepsilon / 2)$ has measure zero we can state the result as follows.

Proposition 3.4 (Demailly). There exists a function $\nu_{\varepsilon}^{j}(\mu, x)$ depending on the eigenvalues of the curvature of $\left(E, h_{\varepsilon}\right)$ which is bounded on compact sets 
of $D$ and right continuous in $\mu$ such that for any $\mu \in \mathbb{R}$

$$
\limsup _{k \longrightarrow \infty} k^{-n} N^{j}\left(\mu, \frac{1}{k} \Delta_{k, \varepsilon}^{\prime \prime}\lceil D(\varepsilon / 2)) \leqslant \frac{1}{n !} \int_{D(\varepsilon / 2)} \nu_{\varepsilon}^{j}(\mu, x) d V(x) .\right.
$$

Moreover there exists an at most countable set $\mathcal{D}_{\varepsilon} \subset \mathbb{R}$ such that for $\mu$ outside $\mathcal{D}_{\varepsilon}$ the limit of the left-hand side expression exists and we have equality in (3.16).

For $\lambda<(1 / 24)$ and sufficiently large $k$ we have

$$
\operatorname{dim} H^{0}\left(D, E^{k}\right) \geqslant N^{0}\left(\lambda, \frac{1}{k} \Delta_{k, \varepsilon}^{\prime \prime}\right)-N^{1}\left(\lambda, \frac{1}{k} \Delta_{k, \varepsilon}^{\prime \prime}\right)
$$

For $\lambda<(1 / 24)$ and $\lambda$ outside $\mathcal{D}_{\varepsilon}$ we apply Proposition 3.4 and Lemma 2.3:

$$
\lim _{k \rightarrow \infty} k^{-n} N^{0}\left(\lambda, \frac{1}{k} \Delta_{k, \varepsilon}^{\prime \prime}\right) \geqslant \frac{1}{n !} \int_{D(\varepsilon / 2)} \nu_{\varepsilon}^{0}(\lambda, x) d V(x) .
$$

On the other hand given $\delta>0$ we learn from Lemma 2.2 that for large $k$

$$
\begin{aligned}
N^{1}\left(\lambda, \frac{1}{k} \Delta_{k, \varepsilon}^{\prime \prime}\right) & \leqslant N^{1}\left(24 \lambda+16 C_{\varepsilon} k^{-1}, \frac{1}{k} \Delta_{k, \varepsilon}^{\prime \prime}\left\lceil_{D(\varepsilon / 2)}\right)\right. \\
& \leqslant N^{1}\left(24 \lambda+\delta, \frac{1}{k} \Delta_{k, \varepsilon}^{\prime \prime}\left\lceil_{D(\varepsilon / 2)}\right)\right.
\end{aligned}
$$

hence

$$
\limsup _{k \rightarrow \infty} k^{-n} N^{1}\left(\lambda, \frac{1}{k} \Delta_{k, \varepsilon}^{\prime \prime}\right) \leqslant \frac{1}{n !} \int_{D(\varepsilon / 2)} \nu_{\varepsilon}^{1}(24 \lambda+\delta, x) d V(x) .
$$

and after letting $k$ go to infinity we can also let $\delta$ go to zero. Using these remarks we see that for all but a countable set of $\lambda$ we have

$$
\liminf _{k \longrightarrow \infty} k^{-n} \operatorname{dim} H^{0}\left(D, E^{k}\right) \geqslant \frac{1}{n !} \int_{D(\varepsilon / 2)}\left[\nu_{\varepsilon}^{0}(\lambda, x)-\nu_{\varepsilon}^{1}(24 \lambda, x)\right] d V(x)
$$

In the latter estimate we may let $\lambda \longrightarrow 0$ (through values outside the exeptional countable set) and this yields, by the formulas in [9] for the right-hand side

$$
\liminf _{k \rightarrow \infty} k^{-n} \operatorname{dim} H^{0}\left(D, E^{k}\right) \geqslant \frac{1}{n !} \int_{D(\varepsilon / 2)\left(\leqslant 1, h_{\varepsilon}\right)}\left(\frac{\imath}{2 \pi} \mathbf{c}\left(E, h_{\varepsilon}\right)\right)^{n}
$$

The set $D(\varepsilon / 2)\left(\leqslant 1, h_{\varepsilon}\right)$ is the set of points in $D(\varepsilon / 2)$ where $\imath \mathbf{c}(E)$ is nondegenerate and has at most one negative eigenvalue. Thus $D(\varepsilon / 2)\left(\leqslant 1, h_{\varepsilon}\right)$ splits in two sets: the set $D(\varepsilon / 2)\left(0, h_{\varepsilon}\right)$ where $\imath \mathbf{c}(E)$ is positive definite 
and the set $D(\varepsilon / 2)\left(1, h_{\varepsilon}\right)$ where $\imath \mathbf{c}(E)$ is non-degenerate and has exactly one negative eigenvalue. The integral in (3.18) splits accordingly into one positive and one negative term:

$$
\begin{aligned}
& \liminf _{k \longrightarrow \infty} k^{-n} \operatorname{dim} H^{0}\left(D, E^{k}\right) \\
& \quad \geqslant \frac{1}{n !} \int_{D(\varepsilon / 2)\left(0, h_{\varepsilon}\right)}\left(\frac{\imath}{2 \pi} \mathbf{c}\left(E, h_{\varepsilon}\right)\right)^{n}+\frac{1}{n !} \int_{D(\varepsilon / 2)\left(1, h_{\varepsilon}\right)}\left(\frac{\imath}{2 \pi} \mathbf{c}\left(E, h_{\varepsilon}\right)\right)^{n} .
\end{aligned}
$$

Our next task is to make $\varepsilon \longrightarrow 0$ in (3.19). For $\varepsilon \longrightarrow 0$ the metrics $h_{\varepsilon}$ converges uniformly to the metric $h$ of positive curvature on every compact set of $D$. So on any compact of $D$ we recover the integral of $\imath \mathbf{c}(E)$. On the other hand $D(\varepsilon / 2)$ exhausts $D$ and the sets $D(\varepsilon / 2)\left(1, h_{\varepsilon}\right)$ concentrate to the boundary $\partial D$.

Let us fix a compact set $L \subset D$. For sufficiently small $\varepsilon$ we have $L \subset$ $D(\varepsilon / 2)$ and

$$
\int_{D(\varepsilon / 2)\left(0, h_{\varepsilon}\right)}\left(\frac{\imath}{2 \pi} \mathbf{c}\left(E, h_{\varepsilon}\right)\right)^{n} \geqslant \int_{L\left(0, h_{\varepsilon}\right)}\left(\frac{\imath}{2 \pi} \mathbf{c}\left(E, h_{\varepsilon}\right)\right)^{n}
$$

We have $h_{\varepsilon} \longrightarrow h$ on $L$ in the $\mathcal{C}^{\infty}$-topology. Since $L(0, h)=L$ letting $\varepsilon \longrightarrow 0$ in the previous inequality yields

$$
\liminf _{\varepsilon \longrightarrow 0} \int_{D(\varepsilon / 2)\left(0, h_{\varepsilon}\right)}\left(\frac{\imath}{2 \pi} \mathbf{c}\left(E, h_{\varepsilon}\right)\right)^{n} \geqslant \int_{L}\left(\frac{\imath}{2 \pi} \mathbf{c}(E, h)\right)^{n}
$$

Let us study the more delicate case of the second integral in (3.19). For this goal we fix on $D$ the ground metric $\omega_{E}=\imath \mathbf{c}(E)$ in order to simplify our computations. We denote by $\lambda_{1}^{\varepsilon} \leqslant \lambda_{2}^{\varepsilon} \leqslant \cdots \leqslant \lambda_{n}^{\varepsilon}$ the eigenvalues of $\imath \mathbf{c}\left(E, h_{\varepsilon}\right)$ with respect to $\omega_{E}$. Then the integral we study is

$$
I_{\varepsilon}=\frac{1}{n !} \int_{D(\varepsilon / 2)\left(1, h_{\varepsilon}\right)}\left(\frac{\imath}{2 \pi} \mathbf{c}\left(E, h_{\varepsilon}\right)\right)^{n}=\frac{1}{(2 \pi)^{n}} \int_{S(\varepsilon)} \lambda_{1}^{\varepsilon} \lambda_{2}^{\varepsilon} \cdots \lambda_{n}^{\varepsilon} \omega_{E}^{n} / n !
$$

where the integration set is

$$
S(\varepsilon):=D(\varepsilon / 2)\left(1, h_{\varepsilon}\right)=\left\{x \in D(\varepsilon / 2): \lambda_{1}^{\varepsilon}(x)<0<\lambda_{2}^{\varepsilon}(x)\right\}
$$

We find an upper bound for $\left|I_{\varepsilon}\right|$ so we determine upper bounds for $\left|\lambda_{1}^{\varepsilon}\right|,\left|\lambda_{2}^{\varepsilon}\right|$, $\ldots,\left|\lambda_{n}^{\varepsilon}\right|$ on $S(\varepsilon)$. Since $\lambda_{1}^{\varepsilon}$ is negative on $S(\varepsilon)$ we have to obtain a lower 
bound for this eigenvalue. By the min-max principle

$$
\lambda_{1}^{\varepsilon}(x)=\min _{v \in T_{x} D} \frac{\left[\imath \mathbf{c}(E, h)+\imath \varepsilon \chi(\varphi) \partial \bar{\partial} \varphi+\imath \varepsilon \chi^{\prime}(\varphi) \partial \varphi \wedge \bar{\partial} \varphi\right](v)}{\imath \mathbf{c}(E)(v)} .
$$

We use now $\imath \varepsilon \chi^{\prime}(\varphi) \partial \varphi \wedge \bar{\partial} \varphi(v) \geqslant 0$. Moreover, since $\lambda_{1}^{\varepsilon}(x)<0$ we have

$$
\min _{v \in T_{x} D} \frac{\imath \partial \bar{\partial} \varphi(v)}{\imath \mathbf{c}(E)(v)}<0, \quad \min _{v \in T_{x} D} \frac{\imath \partial \bar{\partial} \varphi(v)}{\imath \mathbf{c}(E)(v)}=-\max _{v \in T_{x} D} \frac{-\imath \partial \bar{\partial} \varphi(v)}{\imath \mathbf{c}(E)(v)}
$$

Hence

$$
\lambda_{1}^{\varepsilon} \geqslant 1-\varepsilon \chi(\varphi) M_{E}(-\varphi) \quad \text { on } S(\varepsilon) .
$$

The inequality (3.21) gives information about the size of $S(\varepsilon)$. Indeed, $\lambda_{1}^{\varepsilon}<0$ and (3.21) entail $\varphi>-\sqrt{\varepsilon M_{E}(-\varphi)}$. Thus the integration set is contained in a 'corona' of size $\sqrt{\varepsilon}$ :

$$
S(\varepsilon) \subset D(\varepsilon / 2) \bigcap\left\{x \in D: \varphi(x)>-\sqrt{\varepsilon M_{E}(-\varphi)}\right\} .
$$

Since $\varepsilon \chi(\varphi)<2 M_{\omega}^{\prime}(E)$ on $D(\varepsilon / 2)$ (see (3.11)) we deduce the final estimate for the first eigenvalue:

$$
\left|\lambda_{1}^{\varepsilon}\right| \leqslant 2 M_{E}(-\varphi) M_{\omega}^{\prime}(E)-1=: C_{2} \quad \text { on } S(\varepsilon) .
$$

We examine now the eigenvalues $\lambda_{j}^{\varepsilon}$ for $j=2, \ldots, n-1$. The min-max principle yields:

$$
\lambda_{j}^{\varepsilon} \leqslant 1+\varepsilon \chi(\varphi) M_{E}(\varphi)+\min _{\substack{F \subset T_{x} D \\ \operatorname{dim} F=j}} \max _{v \in F} \frac{\imath \varepsilon \chi^{\prime}(\varphi) \partial \varphi \wedge \bar{\partial} \varphi(v)}{\imath \mathbf{c}(E)(v)} .
$$

The minimum in the last expression is 0 and is attained on some space $F \subset$ ker $\partial \varphi$. Therefore we get:

$$
\left|\lambda_{j}^{\varepsilon}\right| \leqslant 1+2 M_{\omega}^{\prime}(E) M_{E}(\varphi)=: C_{3} \quad \text { on } S(\varepsilon) \text { for } j=2, \ldots, n-1
$$

The highest eigenvalue satisfies the estimate:

$$
\lambda_{n}^{\varepsilon} \leqslant 1+\varepsilon \chi(\varphi) M_{E}(\varphi)+\varepsilon \chi^{\prime}(\varphi) \max _{v \in T_{x} D} \frac{\imath \partial \varphi \wedge \bar{\partial} \varphi(v)}{\imath \mathbf{c}(E)(v)} .
$$

The inequalities: $\varepsilon \chi(\varphi)<2 M_{\omega}^{\prime}(E)$ and $\varepsilon \chi^{\prime}(\varphi) \leqslant\left(2 M_{\omega}^{\prime}(E)\right)^{3 / 2} \varepsilon^{-1 / 2}$ hold on 
$D(\varepsilon / 2)$ (the last one since $\left.\chi^{\prime}(\varphi)=-\varphi^{-3}\right)$. We introduce the short notation:

$$
M_{E}^{\varepsilon}(\partial \varphi)=M_{\imath \mathbf{c}(E)}\left(\imath \partial \varphi \wedge \bar{\partial} \varphi, K_{\varepsilon}\right),
$$

where $K_{\varepsilon}:=\bar{D} \backslash\left\{x \in D: \varphi(x)>-\sqrt{\varepsilon M_{E}(-\varphi)}\right\}$. It is clear that $M_{E}^{\varepsilon}(\partial \varphi)$ converges to $M_{E}(\partial \varphi)$ for $\varepsilon \longrightarrow 0$. With this notation,

$$
\left|\lambda_{n}^{\varepsilon}\right| \leqslant 1+2 M_{\omega}^{\prime}(E) M_{E}(\varphi)+\varepsilon^{-1 / 2}\left(2 M_{\omega}^{\prime}(E)\right)^{3 / 2} M_{E}^{\varepsilon}(\partial \varphi) \quad \text { on } S(\varepsilon) .
$$

At this point we may return to $\left|I_{\varepsilon}\right|$ and use the obvious inequality

$$
\left|I_{\varepsilon}\right| \leqslant(2 \pi)^{-n} \operatorname{Vol}_{\mathbf{c}(E)}(S(\varepsilon)) \sup _{S(\varepsilon)}\left|\lambda_{1}^{\varepsilon}\right|\left|\lambda_{2}^{\varepsilon}\right| \cdots\left|\lambda_{n}^{\varepsilon}\right|
$$

where $\operatorname{Vol}_{\mathbf{c}(E)}$ represents the volume with respect to the metric $\imath \mathbf{c}(E)$. We need to find a bound only for the volume. Taking into account (3.22),

$$
\begin{aligned}
& \operatorname{Vol}_{\mathbf{c}(E)}(S(\varepsilon)) \leqslant \sqrt{\varepsilon}\left(\sqrt{M_{E}(-\varphi)}-\sqrt{\left(2 M_{\omega}^{\prime}(E)\right)^{-1}}\right) \\
& \times \sup \left\{\int_{\{\varphi=c\}} \frac{d S_{E}}{|d \varphi|_{E}}: c \in\left[-\sqrt{\varepsilon M_{E}(-\varphi)},-\sqrt{\varepsilon\left(2 M_{\omega}^{\prime}(E)\right)^{-1}}\right]\right\} .
\end{aligned}
$$

Relations (3.25) and (3.26) yield:

$$
\begin{aligned}
& \limsup _{\varepsilon \longrightarrow 0} \operatorname{Vol}_{\mathbf{c}(E)}(S(\varepsilon)) \sup _{S(\varepsilon)}\left|\lambda_{n}^{\varepsilon}\right| \\
& \quad \leqslant\left(\sqrt{2 M_{\omega}^{\prime}(E) M_{E}(-\varphi)}-1\right) 2 M_{\omega}^{\prime}(E) M_{E}(\partial \varphi) \int_{\partial D} \frac{d S_{E}}{|d \varphi|_{E}} \\
& \quad=C_{1} C_{4} \int_{\partial D} \frac{d S_{E}}{|d \varphi|_{E}} .
\end{aligned}
$$

Using (3.23) and (3.24) we conclude

$$
\limsup _{\varepsilon \longrightarrow 0}\left|I_{\varepsilon}\right| \leqslant(2 \pi)^{-n} C_{1} C_{2} C_{3}^{n-2} C_{4} \int_{\partial D} \frac{d S_{E}}{|d \varphi|_{E}} .
$$

We are ready to let $\varepsilon \longrightarrow 0$ in (3.19) and we use (3.20) and (3.27). In (3.20) we can further let the compact $L$ exhaust $D$. This proves (2.1) and with it the Existence Criterion. 


\section{Perturbation of Line Bundles}

In this section we discuss the relation between the perturbation of the complex structure of a line bundle and the perturbation of the complex structure on the base manifold. This requires a glance to the corresponding section of Lempert's article [20]. Let us consider a compact complex manifold $Y=(Y, \mathcal{I})$ with boundary endowed with a complex structure $\mathcal{I}$. Let $Z$ be a smooth divisor in $Y$. Denote as usual by $[Z]$ the associated line bundle. We are interested in the effect of a small perturbation of $\mathcal{I}$ on $Y$ on the complex structure of $[Z]$ or of the canonical bundle $K_{Y}$ over a compact set $D \Subset Y$. This will suffice for the proof of the Stability Theorem. Indeed, denote by $E$ a positive line bundle on a concave manifold $Y$ and assume that for a small perturbation $\mathcal{I}^{\prime}$ of $\mathcal{I}$ there exists a perturbation $E^{\prime}$ of $E$ such that the curvature forms of $E$ and $E^{\prime}$ are close on a sublevel set $D$. Then the right hand-side terms in (2.1) calculated for $\mathcal{I}$ and $\mathcal{I}^{\prime}$ are also close. If one is positive so is the other and both manifolds $D$ and $D^{\prime}$ (and therefore $Y$ and $Y^{\prime}$ ) are Moishezon.

Let us remark that not every perturbation of the complex structure on $Y$ lifts to a perturbation of $[Z]$. We need the hypothesis that the tangent space $T(Z)$ is $\mathcal{I}^{\prime}$ invariant. Then $Z$ is a divisor in the new manifold $Y^{\prime}=\left(Y, \mathcal{I}^{\prime}\right)$ and we consider the associated bundle $[Z]^{\prime}$. Of course any perturbation of $\mathcal{I}$ lifts to a perturbation of the canonical line bundle.

The next Lemma is a "small perturbation" of Lemma 4.1 of Lempert [20]. In the latter a compact divisor $Z \subset \operatorname{Int} Y$ is considered whereas in our case we deal with a divisor which may cut the boundary. However, since we are interested in the effect of the perturbation just on a compact set the proof is the same. We use the $\mathcal{C}^{\infty}$ topology on the spaces of tensors defined on $Y$ and also on spaces of restrictions of tensors to compact subsets of $Y$. We say that two tensors are close when they are close in the $\mathcal{C}^{\infty}$ topology.

Lemma 4.1. Let $(Y, \mathcal{I})$ be a compact complex manifold, $Z$ a smooth divisor in $Y$ and $D \Subset Y$. There exists a finite covering $\mathcal{U}=\left\{U_{\alpha}\right\}_{\alpha \in A}$ of $D$ and a multiplicative cocycle $\left\{g_{\alpha \beta} \in \mathcal{O}_{\mathcal{I}}\left(\bar{U}_{\alpha} \cap \bar{U}_{\beta}\right): \alpha, \beta \in A\right\}$ defining the bundle $E=[Z]$ in the vicinity of $D$, with the following property. If $\mathcal{I}^{\prime}$ is another complex structure on $Y$ close to $\mathcal{I}$ such that $T(Z)$ rests $\mathcal{I}^{\prime}$ invariant, the bundle $E^{\prime}$ determined by $Z$ in the structure $\mathcal{I}^{\prime}$ can be defined in the vicinity of $D$ by the cocycle $\left\{g_{\alpha \beta}^{\prime} \in \mathcal{O}_{\mathcal{I}^{\prime}}\left(\bar{U}_{\alpha} \cap \bar{U}_{\beta}\right): \alpha, \beta \in A\right\}$ such that $g_{\alpha \beta}^{\prime}$ will be 
as close as we please to $g_{\alpha \beta}$ on $\bar{U}_{\alpha} \cap \bar{U}_{\beta}$ assuming $\mathcal{I}^{\prime}$ and $\mathcal{I}$ are sufficiently close.

Proof. We remind for the sake of completeness the construction of the cocycles. For every point of $Y \cap \bar{D}$ there exists an open neighbourhood $U$ in $Y$ and a $\mathcal{I}$-biholomorphism $\psi_{U}$ of some neighbourhood of $\bar{U}$ into $\mathbb{C}^{n}$, $n=\operatorname{dim} Y$, such that $\psi_{U}(U)$ is the unit polydisc and $\psi_{U}(Z) \subset\left\{z \in \mathbb{C}^{n}\right.$ : $\left.z_{1}=0\right\}$. Let $\left\{U_{\alpha}\right\}_{1 \leqslant \alpha \leqslant m}$ be a finite covering consisting of sets $U$ as above and for each $\alpha$ denote by $\psi_{\alpha}$ the corresponding biholomorphism. We select further an open set $U_{0} \Subset Y \backslash Z$ such that $\mathcal{U}=\left\{U_{\alpha}\right\}_{0 \leqslant \alpha \leqslant m}$ is a covering of $\bar{D}$. For every $1 \leqslant \alpha \leqslant m$ we select a smooth strictly pseudoconvex Stein domain $U_{\alpha}^{*} \supset U_{\alpha}$ such that $\psi_{\alpha}$ is biholomorphic in the neighbourhood of $U_{\alpha}^{*}$. Set moreover $U_{0}^{*}=U_{0}$. We construct a cocycle defining $E=[Z]$ in the open set $\cup_{\alpha} U_{\alpha}^{*}$ as follows. First define functions $g_{\alpha}$ such that $g_{0}$ is identically 1 on $U_{0}$ and $g_{\alpha}=z_{1} \circ \psi_{\alpha}$ for $\alpha \geqslant 1$. The bundle $E$ is defined in the vicinity of $D$ by the $\mathcal{I}$ holomorphic multiplicative cocycle $\left\{g_{\alpha \beta}\right\}$ where $g_{\alpha \beta}=g_{\alpha} / g_{\beta}$. Note that $g_{\alpha \beta}$ is holomorphic on a neighbourhood of $\bar{U}_{\alpha}^{*} \cap \bar{U}_{\beta}^{*} \supset \bar{U}_{\alpha} \cap \bar{U}_{\alpha}$.

Let $\mathcal{I}^{\prime}$ be a complex structure as in the statement. Then $Z$ is a complex hypersurface in the new structure and defines a line bundle $E^{\prime}$. We describe next the cocycle of $E^{\prime}$. The hypothesis on the sets $U_{\alpha}^{*}$ allows the use of a theorem of Hamilton [14] for $U_{\alpha}^{*}$. The theorem asserts that for a small perturbation $\mathcal{I}^{\prime}$ of the complex structure on a neighbourhood of $\bar{U}_{\alpha}^{*}$ there is a $\mathcal{I}^{\prime}$ biholomorphism $\psi_{\alpha}^{\prime}$ of a neighbourhood of $\bar{U}_{\alpha}^{*}$ into $\mathbb{C}^{n}$ close to $\psi_{\alpha}$. As shown in [20] we can even assume $\psi_{\alpha}^{\prime}(Z) \subset\left\{z \in \mathbb{C}^{n}: z_{1}=0\right\}$. Set $g_{0}^{\prime}$ to be identically 1 on $U_{0}$ and $g_{\alpha}^{\prime}=z_{1} \circ \psi_{\alpha}^{\prime}$ for $\alpha \geqslant 1$. Then put $g_{\alpha \beta}^{\prime}=g_{\alpha}^{\prime} / g_{\beta}^{\prime}$. Since $\psi_{\alpha}$ and $\psi_{\alpha}^{\prime}$ are close, $g_{\alpha}^{\prime}$ is $\mathcal{I}^{\prime}$ holomorphic on a neighbourhood of $\bar{U}_{\alpha}^{*}$ and $g_{\alpha \beta}^{\prime}$ is $\mathcal{I}^{\prime}$ holomorphic on a neighbourhood of $\bar{U}_{\alpha}^{*} \cap \bar{U}_{\beta}^{*}$. The cocycle $\left\{g_{\alpha \beta}^{\prime}\right\}$ defines $E^{\prime}$ in the open set $\cup_{\alpha} U_{\alpha}^{*}$.

The functions $g_{\alpha}$ and $g_{\alpha}^{\prime}$ are close on $\bar{U}_{\alpha}$. We can now repeat the arguments from [20] to show that $g_{\alpha \beta}$ and $g_{\alpha \beta}^{\prime}$ are also close on $\bar{U}_{\alpha} \cap \bar{U}_{\beta}$.

Lemma 4.2. Let $(Y, \mathcal{I}), Z$ and $D \Subset Y$ be as in the preceding Lemma. Assume that $[Z]$ is endowed with a hermitian metric $h$. If $\mathcal{I}^{\prime}$ is another complex structure on $Y$ close to $\mathcal{I}$, leaving $T(Z)$ invariant, there exists a hermitian metric $h^{\prime}$ on the line bundle $[Z]^{\prime}$ near $D$ such that the curvature form $\imath \mathbf{c}\left([Z]^{\prime}\right)$ will be as close as we please to $\imath \mathbf{c}([Z])$ on $D$ assuming $\mathcal{I}^{\prime}$ and $\mathcal{I}$ are sufficiently close. 
Proof. We can define a smooth bundle isomorphism $[Z] \longrightarrow[Z]^{\prime}$ in the vicinity of $D$ by resolving the smooth additive cocycle $\log \left(g_{\alpha \beta}^{\prime} / g_{\alpha \beta}\right)$ in order to find smooth functions $f_{\alpha}$, close to 1 on a neighbourhood of $\bar{U}_{\alpha}$ such that $g_{\alpha \beta}^{\prime}=f_{\alpha} g_{\alpha \beta} f_{\beta}^{-1}$. Then the isomorphism between $[Z]$ and $[Z]^{\prime}$ is defined by $f=\left\{f_{\alpha}\right\}$. The metric $h$ is given in terms of the covering $\mathcal{U}$ by a collection $h=\left\{h_{\alpha}\right\}$ of smooth strictly positive functions satisfying the relation $h_{\beta}=$ $h_{\alpha}\left|g_{\alpha \beta}\right|$. We define a hermitian metric $h^{\prime}=\left\{h_{\alpha}^{\prime}\right\}$ on $[Z]^{\prime}$ by $h_{\alpha}^{\prime}=h_{\alpha}\left|f_{\alpha}^{-1}\right|$; $h_{\alpha}^{\prime}$ is close to $h_{\alpha}$ on $D$. The curvature form of $[Z]^{\prime}$ has the form

$$
\frac{\imath}{2 \pi} \mathbf{c}\left([Z]^{\prime}\right)=\frac{1}{4 \pi} d \circ \mathcal{I}^{\prime} \circ d\left(\log h_{\alpha}^{\prime}\right) .
$$

Therefore, when $\mathcal{I}^{\prime}$ is sufficiently close to $\mathcal{I}, \frac{l}{2 \pi} \mathbf{c}\left([Z]^{\prime}\right)$ is close to $\frac{\imath}{2 \pi} \mathbf{c}([Z])$ on $D$.

In the same vein we study the perturbation of the canonical bundle.

Lemma 4.3. Let $(Y, \mathcal{I})$ and $D \Subset Y$ be as above. Assume $K_{Y}$ is endowed with a hermitian metric $h$. If $\mathcal{I}^{\prime}$ is another complex structure on $Y$ close to $\mathcal{I}$, there exists a hermitian metric $h^{\prime}$ on $K_{Y^{\prime}}$ near $D$ such that the curvature form $\imath \mathbf{c}\left(K_{Y^{\prime}}\right)$ will be as close as we please to $\imath \mathbf{c}\left(K_{Y}\right)$ on $D$ assuming $\mathcal{I}^{\prime}$ and $\mathcal{I}$ are sufficiently close.

Proof. We find as before a finite covering $\mathcal{U}=\left\{U_{\alpha}\right\}_{\alpha \in A}$ of $\bar{D}$ and biholomorphisms $\psi_{\alpha}$ defined in a neighbourhood of $\bar{U}_{\alpha}$ which map $U_{\alpha}$ onto the unit polydisc in $\mathbb{C}^{n}$. For every $\alpha \in A$ we select a smooth strictly pseudoconvex Stein domain $U_{\alpha}^{*} \supset U_{\alpha}$ such that $\psi_{\alpha}$ is biholomorphic in the neighbourhood of $U_{\alpha}^{*}$. The canonical bundle $K_{Y}$ is defined in the vicinity of $D$ by $g_{\alpha \beta}=\operatorname{det}\left(\partial \psi_{\alpha} / \partial \psi_{\beta}\right)=\operatorname{det}\left(\partial\left(\psi_{\alpha} \circ \psi_{\beta}^{-1}\right) / \partial w\right)$ which is $\mathcal{I}$-holomorphic on a neighbourhood of $\bar{U}_{\alpha}^{*} \cap \bar{U}_{\beta}^{*} \supset \bar{U}_{\alpha} \cap \bar{U}_{\alpha}$. Here $w$ are the canonical coordinates on $\mathbb{C}^{n}$. We apply as before Hamilton's theorem and obtain $\mathcal{I}^{\prime}$ biholomorphisms $\psi_{\alpha}^{\prime}$ in a neighbourhood of $\bar{U}_{\alpha}^{*}$ into $\mathbb{C}^{n}$ close to $\psi_{\alpha}$.

The cononical bundle $K_{Y^{\prime}}$ is defined in the vicinity of $D$ by $g_{\alpha \beta}^{\prime}=$ $\operatorname{det}\left(\partial \psi_{\alpha}^{\prime} / \partial \psi_{\beta}^{\prime}\right)$. Since $\psi_{\alpha}^{\prime}$ is close to $\psi_{\alpha}$ we see that $g_{\alpha \beta}^{\prime}$ is close to $g_{\alpha \beta}$ on $\bar{U}_{\alpha} \cap \bar{U}_{\alpha}$. By repeating the arguments in the proof of Lemma 4.2 we conclude. 


\section{The Stability Theorem}

In this section we prove the Stability Theorem. Let us consider a compact manifold $M, \operatorname{dim} M=n$, and a complex submanifold $A$ of dimension $q$. Then $X=M \backslash A$ is $(q+1)$-concave. Let us remind the construction of an exhaustion function. Select a finite covering $\hat{\mathcal{U}}=\left\{U_{\alpha}\right\}_{\alpha} \geqslant 1$ of $A$ with coordinate domains such that if the coordinates in $U_{\alpha}$ are $z_{\alpha}=\left(z_{\alpha}^{1}, z_{\alpha}^{2}, \cdots, z_{\alpha}^{n}\right)$ we have $A \cap U_{\alpha}=\left\{z \in U_{\alpha}: z_{\alpha}^{q+1}=\cdots=z_{\alpha}^{n}=0\right\}$. Set $\varphi_{\alpha}(z)=\sum_{q+1}^{n}\left|z_{\alpha}^{j}\right|^{2}$. Choose a relatively compact open set $U_{0} \Subset M \backslash A$ such that $\mathcal{U}=\left\{U_{0}\right\} \cup \hat{\mathcal{U}}=\left\{U_{\alpha}\right\}_{\alpha \geqslant 0}$ is a covering of $M$ and set $\varphi_{0} \equiv 1$ on $U_{0}$. Let $\left\{\rho_{\alpha}\right\}_{\alpha \geqslant 0}$ be a partition of unity subordinated to $\mathcal{U}$. Define $\varphi=\varphi_{A}=\sum_{\alpha \geqslant 0} \rho_{\alpha} \varphi_{\alpha}$. The function $\varphi$ enjoys the following properties:

1. $\varphi \in \mathcal{C}^{\infty}(M), A=\{\varphi=0\}$ and $\varphi \geqslant 0$.

2. For any $c>0$ we have $\{\varphi>c\} \Subset M \backslash A$.

3. $\partial \bar{\partial} \varphi=\sum_{\alpha}\left(\rho_{\alpha} \partial \bar{\partial} \varphi_{\alpha}+\varphi_{\alpha} \partial \bar{\partial} \rho_{\alpha}+\partial \rho_{\alpha} \wedge \bar{\partial} \varphi_{\alpha}+\partial \varphi_{\alpha} \wedge \bar{\partial} \rho_{\alpha}\right)$ where $\partial \bar{\partial} \varphi_{\alpha}=2 \sum_{q+1}^{n} d z_{\alpha}^{j} \wedge d \bar{z}_{\alpha}^{j}$.

For $z \in A, \partial \bar{\partial} \varphi(z)=\sum_{\alpha} \rho_{\alpha}(z) \partial \bar{\partial} \varphi_{\alpha}(z)$ has $n-q$ positive eigenvalues. Hence $\partial \bar{\partial} \varphi$ has $n-q$ positive eigenvalues in a neighbourhood of $A$. Moreover $\partial \bar{\partial} \varphi$ is positive semidefinite on $A$. Let us construct a hermitian metric on $M$ which is "small" in the normal direction to $A$ (near $A$ ) and "large" in the tangential direction to $A$. We can consider on each $U_{\alpha}$ the metric $\delta^{-1} \sum_{1}^{q} d z_{\alpha}^{j} \wedge d \bar{z}_{\alpha}^{j}+\delta \sum_{q+1}^{n} d z_{\alpha}^{j} \wedge d \bar{z}_{\alpha}^{j},(\delta>0)$, and then patch these metrics together with the partition of unity to obtain a metric $\omega_{\delta}$ on $M$. Let $\gamma_{1}^{\delta} \leqslant$ $\gamma_{2}^{\delta} \leqslant \cdots \leqslant \gamma_{n}^{\delta}$ be the eigenvalues of $\imath \partial \bar{\partial} \varphi$ with respect to $\omega_{\delta}$. For $\delta$ sufficiently small there exists a neighbourhood $U_{\delta}$ of $A$ such that on $U_{\delta}, \gamma_{j}^{\delta} \geqslant-O(\delta)$ for $j=1, \ldots, q$ and $\gamma_{j}^{\delta} \geqslant O\left(\delta^{-1}\right)$ for $j=q+1, \ldots, n$. Therefore we can choose $\delta$ such that on $U_{\delta}, \gamma_{j}^{\delta} \geqslant-1$ for $j=1, \ldots, q$ and $\gamma_{j}^{\delta} \geqslant 2 n-3$ for $j=q+1, \ldots, n$.

Let us consider now the domains $X_{c}=\{\varphi>c\}$ for $c>0$ sufficiently small. If $\operatorname{codim} A \geqslant 3$ the domains $X_{c}$ admit as definition function $c-\varphi$ whose complex hessian has 3 negative eigenvalues in the vicinity of $\partial X_{c}$. If $M$ possesses a positive line bundle we are in the conditions of the Existence Criterion. Note that the metric $\omega_{\delta}$ satisfies Property 2.2 for all $X_{c}$ with $c$ sufficiently small. For technical reasons we construct a metric $\omega$ as follows. 
Consider the real part $g_{\delta}$ of the hermitian metric $\omega_{\delta}$. Thus $g_{\delta}$ is a riemannian metric on $M$. Take a hermitian metric $\omega$ whose real part $g$ satisfies $g(u, v)=$ $g_{\delta}(u, v)+g_{\delta}(\mathcal{I} u, \mathcal{I} v)(u, v \in \mathbb{C} \otimes T(M))$ where $\mathcal{I}$ is the complex structure of $M$. If $\delta$ is sufficiently small $\omega$ still satisfies Property 2.2. From now on we fix such a metric $\omega$ on $M$. The constants $M_{\omega}^{\prime}(E)$ are calculated with respect to this metric.

Lemma 5.1. Assume that $M$ is a projective manifold and $E$ is a positive line bundle over $M$. Let $A$ be a submanifold with $\operatorname{codim} A \geqslant 3$. Then for sufficiently small regular values $c>0$ we have

$$
\int_{X_{C}}\left(\frac{\imath}{2 \pi} \mathbf{c}(E)\right)^{n}>C(c-\varphi, E) \int_{\partial X_{c}} \frac{d S_{E}}{|d \varphi|_{E}}
$$

where $C(c-\varphi, E)$ has been introduced in (3.1).

Proof. Remark first that the constant $C(c-\varphi, E)$ converges to 0 for $c \longrightarrow 0$. Indeed, $\partial \bar{\partial}(c-\varphi)=-\partial \bar{\partial} \varphi$ so the constants $M_{E}(c-\varphi), M_{E}(\varphi-c)$ and $M_{\omega}^{\prime}(E)$ are bounded for $c$ running in a compact interval since $\partial \bar{\partial} \varphi$ and $E$ are defined over all $M$. We observe further that $d \varphi(z) \longrightarrow 0$ when $z \longrightarrow A$ (in fact $\left.d \varphi \uparrow_{A}=0\right)$. Hence $M_{E}\left(\partial(c-\varphi) \wedge \bar{\partial}(c-\varphi), \partial X_{c}\right)$ converges to 0 (and with it $C(c-\varphi))$ when $c$ goes to 0 . Examine now the term

$$
\int_{\partial X_{c}} \frac{d S_{E}}{|d \varphi|_{E}}
$$

Although $\left|d \varphi_{E}\right| \longrightarrow 0$ for $z \longrightarrow A$ this integral goes to 0 too for $c \longrightarrow 0$. Indeed, since $A$ has codimension $\geqslant 3$ we have

$$
\int_{\partial X_{c}} d S_{E}=\int_{\{\varphi=c\}} d S_{E}=O\left(c^{5}\right), \quad c \longrightarrow 0 .
$$

On the other hand for a regular value $c$ of $\varphi$,

$$
|d \varphi|_{\partial X_{c}} \mid=O(c), \quad c \longrightarrow 0
$$

We infer

$$
\int_{\partial X_{c}} \frac{d S_{E}}{|d \varphi|_{E}}=O\left(c^{4}\right), \quad c \longrightarrow 0
$$


for regular values $c$ of $\varphi$. In conclusion the boundary integral in (5.1) goes to 0 as $c \longrightarrow 0$. The domain integral in (5.1) being bounded from below by a positive constant the Lemma follows.

At this stage we can prove the Stability Theorem. Let us consider a smooth domain $Y:=X_{c}$ for $c$ small enough such that condition (5.1) holds. Let $\mathcal{I}^{\prime}$ be a new complex structure on $Y$ which leaves $T(Z)$ invariant, for an ample smooth divisor $Z$ on $M$. We apply Lemma4.2 for the manifold $Y$ and a smooth relatively compact set $\bar{D}$ where $D:=X_{d}, d>c$, such that (5.1) still holds on $X_{d}$. By hypothesis the bundle $E$ carries a hermitian metric with positive curvature. Lemma 4.2 shows that there exists a hermitian metric $h^{\prime}$ on the bundle $E^{\prime}$ near $D$ such that $\imath \mathbf{c}(E)$ and $\imath \mathbf{c}\left(E^{\prime}\right)$ are as close as we please in the $\mathcal{C}^{\infty}$ topology on $\bar{D}$ if $\mathcal{I}$ and $\mathcal{I}^{\prime}$ are sufficiently close. In particular $\imath \mathbf{c}\left(E^{\prime}\right)$ is positive near $\bar{D}$. Note that a defining function for $D^{\prime}$ is still $d-\varphi$ and its complex hessian will have 3 negative eigenvalues in the vicinity of $\partial D^{\prime}$ for a small perturbation of the complex structure.

Thus we can apply the Existence Criterion for $D^{\prime}$ and $E^{\prime}$. In order to calculate the constant $C\left(d-\varphi, E^{\prime}\right)$ we construct first a metric $\omega^{\prime}$ on $Y$ in the following way. The metric $\omega$ determines a riemannian metric $g$ on $Y$ which was chosen such that $g(u, v)=g_{\delta}(u, v)+g_{\delta}(\mathcal{I} u, \mathcal{I} v)$ for $u, v \in \mathbb{C} \otimes T(M)$. We consider then a hermitian metric $\omega^{\prime}$ on $Y^{\prime}$ with real part $g^{\prime}$ where $g^{\prime}(u, v)=$ $g_{\delta}(u, v)+g_{\delta}\left(\mathcal{I}^{\prime} u, \mathcal{I}^{\prime} v\right)$ for $u, v \in \mathbb{C} \otimes T(M)$. The metric $\omega^{\prime}$ satisfies the Property 2.2 with respect to the defining function $d-\varphi$ of $D^{\prime}$, provided $\mathcal{I}$ and $\mathcal{I}^{\prime}$ are sufficiently close. Therefore the constants $M_{E^{\prime}}(d-\varphi), M_{E^{\prime}}(\varphi-d)$, $M_{\omega^{\prime}}\left(E^{\prime}\right)$ and $M_{E^{\prime}}\left(\partial(d-\varphi), \partial D^{\prime}\right)$ are close to the corresponding constants $M_{E}(d-\varphi), M_{E}(\varphi-d), M_{\omega}(E)$ and $M_{E}(\partial(d-\varphi), \partial D)$ respectively. This entails that $C\left(d-\varphi, E^{\prime}\right)$ is close to $C(d-\varphi, E)$.

It is also clear that $\int_{D^{\prime}}\left(\frac{\imath}{2 \pi} \imath \mathbf{c}\left(E^{\prime}\right)\right)^{n}$ and $\int_{\partial D^{\prime}} d S_{E^{\prime}} /|d \varphi|_{E^{\prime}}$, are close to the corresponding integrals on $D$ and $\partial D$ of $\frac{\imath}{2 \pi} \imath \mathbf{c}(E)$ and $d S_{E^{\prime}} /|d \varphi|_{E^{\prime}}$. Therefore

$$
\int_{D^{\prime}}\left(\frac{\imath}{2 \pi} \mathbf{c}\left(E^{\prime}\right)\right)^{n}>C\left(d-\varphi, E^{\prime}\right) \int_{\partial D^{\prime}} \frac{d S_{E^{\prime}}}{|d \varphi|_{E^{\prime}}} .
$$

By the Existence Criterion

$$
\operatorname{dim} H^{0}\left(D^{\prime}, E^{\prime k}\right) \gtrsim k^{n}
$$


for large $k$ and thus $D^{\prime}$ and so $Y^{\prime}$ are Moishezon, provided $\mathcal{I}$ and $\mathcal{I}^{\prime}$ are sufficiently close. An entirely analogous argument takes care of the case of perturbation of the canonical bundle $K_{Y}$. This proves the Stability Theorem.

\section{Acknowledgments}

A version of the existence criterion for holomorphic sections was obtained in a joint manuscript with Gennadi Henkin, using the original method of Siu [27]. I wish to dedicate this paper to the memory of Gennadi Henkin. The intense intellectual atmosphere of his working group at the University Paris 6 is unforgettable. I would like to thank Prof. Chin-Yu Hsiao for hospitality at Academia Sinica during my visit in December 2015.

\section{References}

1. A. Andreotti, Théorèmes de dépendance algébrique sur les espaces complexes pseudoconcaves, Bull. Soc. Math. France, 91 (1963), 1-38.

2. A. Andreotti and H. Grauert, Théorème de finitude pour la cohomologie des espaces complexes, Bull. Soc. Math. France, 90 (1962), 193-259.

3. A. Andreotti and F. Norguet, Problème de Levi et convexité holomorphe pour les classes de cohomologie, Ann. Scuola Norm. Sup. Pisa (3), 20 (1966), 197-241.

4. A. Andreotti and G. Tomassini, Some remarks on pseudoconcave manifolds, Essays on Topology and Related Topics dedicated to G. de Rham, A. Haefinger and R. Narasimhan Eds., Springer Verlag, 1970, 85-104.

5. A. Andreotti and Y. T. Siu, Projective embeddings of pseudoconcave spaces, Ann. Scuola Norm. Sup. Pisa Cl. Sci. (3), 24 (1970), 231-278.

6. A. Andreotti and E. Vesentini, Carleman estimates for the Laplace-Beltrami equation on complex manifolds, Inst. Hautes Etudes Sci. Publ. Math. No., 25 (1965), 81-130.

7. R. Berman, Holomorphic Morse inequalities on manifolds with boundary, Ann. Inst. Fourier (Grenoble), 55 (2005), no. 4, 1055-1103.

8. T. Bouche, Inegalités de Morse pour la $d^{\prime \prime}$-cohomologie sur une variété non-compacte, Ann. Sci. Ecole Norm. Sup., 22 (1989), 501-513.

9. J. P. Demailly, Champs magnétiques et inegalités de Morse pour la $d^{\prime \prime}$-cohomologie, Ann. Inst. Fourier, 35 (1985), no. 4, 189-229.

10. J. P. Demailly, Estimations $L^{2}$ pour l'opérateur $\bar{\partial}$ d'un fibré vectoriel holomorphe semi-positif au-dessus d'une variété Kählérienne complète, Ann. Sci. École Norm. Sup., 15 (1982), 457-511. 
11. C. Epstein and G. Henkin, Stability of embeddings for pseudoconcave surfaces and their boundaries, Acta Math., 185 (2000), 161-237.

12. E. Getzler, An analoque of Demailly's inequality for strictly pseudoconvex CR manifolds, J. Differential Geom., 29 (1989), no. 2, 231-244.

13. Ph. A. Griffiths, The extension problem in complex analysis, II; Embeddings with positive normal bundle, Amer. J. Math., 88 (1966), 366-446.

14. R. S. Hamilton, Deformation of complex structures on manifolds with boundary. I, $J$. Differential Geom., 12 (1977), 1-45.

15. G. Henkin and J. Leiterer, Andreotti-Grauert theory by integral formulas, Progress in Mathematics, vol. 74, Birkhäuser Boston, Inc., Boston, MA, 1988.

16. G. Henniart, Les inégalités de Morse (d'après Witten), Astérisque, no. 121/122, (1983/84), 43-61, 1985.

17. C-Y. Hsiao and X. Li, Szegö kernel asymptotics and Morse inequalities on CR manifolds with $S^{1}$-action, arXiv:1502.02365.

18. C.-Y. Hsiao and X. Li, Morse inequalities for Fourier components of Kohn-Rossi cohomology of CR manifolds with $S^{1}$-action, Math. Z. (2016), doi:10.1007/s00209-0161661-6.

19. C-Y. Hsiao and G. Marinescu, Szegö kernel asymptotics and Morse inequalities on CR manifolds, Math. Z. 271 (2012), 509-553.

20. L. Lempert, Embeddings of three dimensional Cauchy-Riemann manifolds, Math. Ann., 300 (1994), 1-15.

21. X. Ma and G. Marinescu, Holomorphic Morse inequalities and Bergman kernels, Progress in Math., vol. 254, Birkhäuser, Basel, 2007, 422 pp.

22. G. Marinescu, Morse inequalities for q-positive line bundles over weakly 1-complete manifolds, C. R. Acad. Sci. Paris Sér. I Math., 315 (1992), no. 8, 895-899.

23. G. Marinescu, Asymptotic Morse inequalities for pseudoconcave manifolds, Ann. Scuola Norm. Sup. Pisa Cl. Sci. (4), 23 (1996), no. 1, 27-55.

24. A. Nadel and H. Tsuji, Compactification of complete Kähler manifolds of negative Ricci curvature, J. Differential Geom., 28 (1988), no. 3, 503-512.

25. T. Ohsawa, Isomorphism theorems for cohomology groups of weakly 1-complete manifolds, Publ. Res. Inst. Math. Sci., 18(1982), 191-232.

26. Partial differential equations. VII, Encyclopaedia of Mathematical Sciences, 64, Spectral theory of differential operators, A translation of Current problems in mathematics. Fundamental directions. Vol. 64 (Russian), Akad. Nauk SSSR, Vsesoyuz. Inst. Nauchn. i Tekhn. Inform., Moscow, 1989, Translation by T. Zastawniak, Translation edited by M. A. Shubin, Springer-Verlag, Berlin, 1994.

27. Y. T. Siu, A vanishing theorem for semipositive line bundles over non-Kähler manifolds, J. Differential Geom., 20 (1984), 431-452.

28. R. Todor, I. Chiose and G. Marinescu, Asymptotic morse inequalities for covering manifolds, Nagoya Math. J., 163 (2001), 145-165. 